\title{
Implante de válvulas transcatéter en prótesis valvulares quirúrgicas disfuncionantes: un cambio de paradigma
}

\author{
Dr. Gustavo Vignolo
}

\section{Resumen}

La disfunción de las bioprótesis quirúrgicas frecuentemente ocurre en pacientes añosos, con severas comorbilidades, lo que implica un riesgo elevado frente a una nueva cirugía valvular. El implante de válvulas transcatéter dentro de bioprótesis disfuncionantes o procedimiento valve in valve (VIV), ha sido desarrollado como una nueva alternativa terapéutica para este tipo de situación. Los procedimientos VIV deben ser considerados para pacientes con disfunción protésica severa sintomática con alto riesgo quirúrgico, luego de un exhaustivo análisis individualizado por un grupo de expertos o heart team. La selección y evaluación previa de los pacientes son fundamentales para el éxito del procedimiento. La evidencia respalda más fuertemente este tipo de intervención en posición aórtica que en posición mitral. En esta revisión analizamos brevemente las indicaciones, la evaluación previa de los pacientes, los aspectos relevantes del procedimiento y sus complicaciones eventuales, con especial énfasis en el procedimiento VIV mitral, que ha sido motivo de dos recientes reportes de casos en esta revista.

Palabras clave: $\quad$ PRÓTESIS VALVULARES CARDÍACAS

IMPLANTACIÓN DE PRÓTESIS DE VÁLVULAS CARDÍACAS

VALVE IN VALVE MITRAL TRANSCATÉTER

\section{Transcatheter valve implantation in dysfunctioning surgical valvular prostheses: a paradigm shift}

\section{Summary}

Bioprosthetic surgical valve dysfunction occurs frequently in elderly patients with severe comorbidities, which implies a high surgical risk in case of redo valve surgery. Transcatheter valve implantation within a surgical bioprosthesis, so-called valve in valve procedure (VIV), has been developed as new treatment option for this situation. VIV procedures should be considered in patients with severely symptomatic prosthetic valve dysfunction with high surgical risk, after careful heart team evaluation. Patient selection and pre-procedural evaluation are mainstay for procedural success. Current evidence has stronger support for VIV procedure in aortic than in mitral position. In this review, we briefly analyze VIV procedure indications, patient evaluation, as well as relevant aspects of the procedure itself and its most frequent complications. Special emphasis will be given for VIV in mitral position which has been the focus of two recent case reports in this journal.

Key words: $\quad$ HEART VALVE PROSTHESIS

HEART VALVE PROSTHESIS IMPLANTATION

TRANSCATHETER MITRAL VALVE-IN-VALVE

Médico cardiólogo e intensivista.Centro Cardiovascular Universitario, Hospital de Clínicas. Facultad de Medicina, Universidad de la República.

Correspondencia: Dr. Gustavo Vignolo. Correo electrónico: gvignolo@gmail.com

Recibido Oct 30, 2017; aceptado Nov 2, 2017 
Las bioprótesis quirúrgicas son frecuentemente implantadas durante procedimientos de cirugía valvular mitral. La ventaja de las bioprótesis sobre las prótesis mecánicas es que hacen innecesaria la anticoagulación y por tanto evitan el riesgo de sangrado que esta conlleva. En contraste, las bioprótesis son más susceptibles a la falla estructural con el transcurso del tiempo y esto aumenta la necesidad de reintervención ${ }^{(1)}$.

La sobrevida libre de reoperación por degeneración de bioprótesis es de aproximadamente $95 \%$, $90 \%$ y $70 \%$ a 5,10 y 15 años, respectivamente ${ }^{(2)}$.

Es por esto que tradicionalmente las bioprótesis han sido usadas en pacientes añosos, en los que el riesgo de sangrado es elevado y la expectativa de durabilidad de la bioprótesis es mayor que la expectativa de vida del paciente.

Las recomendaciones actuales de la Sociedad Europea de Cardiología (ESC) sobre el manejo de la enfermedad valvular destacan que la elección entre prótesis mecánica y biológica debe ser multifactorial. Los factores que favorecen el implante de una bioprótesis, incluyen, con nivel de recomendación IC, la opinión informada del paciente, la baja probabilidad de lograr una anticoagulación correcta, el elevado riesgo de sangrado y el antecedente de trombosis de una prótesis mecánica bajo correcta anticoagulación. Como recomendaciones IIa C, se debe considerar el implante de bioprótesis cuando la probabilidad de reintervención valvular o el riesgo de la misma es bajo, así como en pacientes mayores de 65 años para prótesis en posición aórtica y mayores de 70 años en posición mitral, o en aquellos en los que la expectativa de vida es menor que la durabilidad prevista de la bioprótesis. En los pacientes entre 65 y 70 años candidatos a prótesis mitral y entre 60 y 65 años candidatos a prótesis aórtica, ambos tipos de prótesis, biológicas y mecánicas, son aceptables y la elección debe basarse en los demás criterios men$\operatorname{cionados}^{(3)}$. Las variables edad y durabilidad de la prótesis son de la mayor importancia. La edad promedio de la población está aumentando, al igual que la durabilidad de las bioprótesis, por lo cual el número de bioprótesis implantadas también está en aumento, especialmente en posición mitral $^{(4)}$. Esto determina un incremento del número de casos de disfunción protésica en pacientes añosos, lo que constituye un problema clínico emergente. La cirugía de reintervención valvular es el tratamiento estándar de la disfunción protésica severa sintomática. La mortalidad asociada a reintervención por cirugía valvular ha disminuido en las últimas décadas en pacientes de bajo riesgo quirúrgico, de tal manera que en la reinter- vención por cirugía valvular aórtica de coordinación se han reportado valores de $2 \%$ a $7 \%$. Es por esto que en pacientes de bajo riesgo la reintervención sigue siendo el procedimiento de elección ${ }^{(5,6)}$. Sin embargo, la mortalidad puede alcanzar el 30\% en pacientes de alto riesgo y en cirugía no electi$\mathrm{va}^{(7)}$.

Esto representa un desafío terapéutico porque los pacientes con disfunción protésica son frecuentemente añosos, frágiles, con disfunción sistólica o comorbilidades severas, lo que aumenta aun más el riesgo de una eventual reintervención quirúrgica.

Por otra parte, algunas condiciones comórbidas, cuya prevalencia aumenta con la edad, se asocian con necesidad de reintervención por degeneración de bioprótesis, como diabetes mellitus, falla renal, insuficiencia cardíaca congestiva, hipertensión pulmonar y enfermedad coronaria y arterial periférica. Estas variables se asocian también con morbimortalidad operatoria(8)

Implante de prótesis valvulares transcatéter dentro de prótesis valvulares biológicas quirúrgicas: procedimiento valve-in-valve

El desarrollo tecnológico de las prótesis transcatéter y de sus sistemas de implante, asociado a la necesidad de soluciones de menor riesgo que el asociado a cirugía, ha aumentado el espectro de las indicaciones potenciales de este tipo de procedimientos. El implante transcatéter de prótesis valvulares en válvulas nativas, específicamente en posición aórtica (TAVI), lleva 15 años de desarrollo y representa una revolución en la cardiología intervencionista, comparable con la causada por el desarrollo de los stents coronarios. Desde el primer procedimiento de TAVI, realizado por Alain Cribier en 2002, esta tecnología se ha expandido a indicaciones adicionales como el implante de prótesis valvulares transcatéter dentro de prótesis valvulares quirúrgicas disfuncionantes, procedimiento conocido como valve in valve (VIV) y dentro de anillos protésicos (valve in ring o VIR).

En el número anterior de la Revista Uruguaya de Cardiología (RUC) se presentaron dos casos de VIV mitral. Se trata de la comunicación de una experiencia pionera en Latinoamérica sobre este tipo de procedimientos por un grupo de cardiólogos procedentes de México y otro de nuestro país ${ }^{(9,10)}$.

Esta breve revisión se centrará en el procedimiento VIV mitral (VIVM).

Como en la TAVI para la estenosis aórtica nativa, la selección de pacientes es crítica para el éxito de este tipo de procedimientos y es imprescindible 
contar con un equipo especializado en el tema (heart team), que deberá incluir cirujanos cardíacos, cardiólogos intervencionistas, cardiólogos clínicos, imagenólogos cardiovasculares y anestesiólogos.

Las guías para el manejo de la enfermedad valvular cardíaca de la ESC incluyen el procedimiento VIV entre sus recomendaciones para el tratamiento de las prótesis biológicas disfuncionantes: el procedimiento VIV transcatéter en posición aórtica debe ser considerado por el heart team dependiendo del riesgo de la reintervención y del tipo y tamaño de la prótesis (recomendación IIa, nivel de evidencia C). Se reconoce el procedimiento VIV como una opción para el tratamiento de bioprótesis disfuncionantes en pacientes con riesgo quirúrgico aumentado, debiendo analizarse cada caso por parte del heart team en forma individualizada. De todas formas, la evidencia avala el procedimiento en posición aórtica con mayor peso que para la posición mitral, para la cual no hay recomendaciones específicas de la guía referida. Asimismo, se contraindican los procedimientos percutáneos con balón (valvuloplastia) para el tratamiento de estenosis de bioprótesis aórticas o mitrales, aunque no es claro si esto se refiere a procedimientos puros con balón o a valvuloplastia previa al implante de VIV, lo cual es un punto de discusión entre los autores referentes. En general, se desaconseja la valvuloplastia previa al procedimiento VIV, salvo en casos de estenosis bioprotésica muy severa $^{(3)}$.

En resumen, los procedimientos VIV deben ser considerados para pacientes con disfunción protésica severa sintomática con alto riesgo quirúrgico luego de un exhaustivo análisis individualizado por un grupo de expertos o heart team.

\section{Evidencia sobre el procedimiento VIVM}

El primer procedimiento de tipo VIVM en humanos fue realizado en 2009 por Cheung y colabora$\operatorname{dores}^{(11)}$.

En una serie de 23 pacientes consecutivos con disfunción bioprotésica mitral severa tratados con VIVM por acceso transapical, la disfunción protésica que motivó el procedimiento fue estenosis en $26,1 \%$, insuficiencia en $39,1 \%$ y combinada en $34,8 \%$ de los pacientes. Todos los pacientes eran añosos (edad media $81 \pm 6$ años) y presentaban alto riesgo frente a una nueva cirugía convencional para recambio protésico (score STS medio: $12,1 \pm 6,8 \%$ ). El procedimiento fue exitoso en el $100 \%$ de los casos con prótesis expandibles por balón (Sapien, Edwards Lifesciences, Irvine, Cali- fornia) sin complicaciones mayores intraprocedimiento. Durante la internación se reportó un stroke mayor $(4,4 \%)$ y seis sangrados mayores $(26,1 \%)$. El gradiente transvalvular mitral disminuyó significativamente de $11,1 \pm 4,6 \mathrm{mmHg}$ a $6,9 \pm 2,2 \mathrm{mmHg}$ luego del procedimiento $(\mathrm{p}<0,01)$. No se comprobó insuficiencia intravalvular en ningún caso. La sobrevida a 30 días fue de $100 \%$ y de $90,4 \%$ al fin del período de seguimiento (753 días). Todos los pacientes vivos estaban en clase funcional NYHA I-II con sus respectivas prótesis transcatéter normofuncionantes ${ }^{(12)}$.

La factibilidad del implante de la prótesis expandible por balón Inovare Braile fue documentada en una serie de 12 casos realizados por acceso transapical. La edad media de la serie fue de 61,6 $\pm 9,9$ años, $92 \%$ fueron pacientes de sexo femenino, con un Euroscore logístico medio de 20,1\%. El implante fue exitoso en todos los casos, aunque en uno ocurrió embolización de la prótesis que requirió cirugía para su remoción. No hubo mortalidad en el procedimiento y la sobrevida a 30 días fue de $91,7 \%$. Se comprobó una reducción significativa del gradiente transmitral (11 mmHg vs $6 \mathrm{mmHg}$; $<0,001$ ), no observándose insuficiencia mitral residual ni obstrucción del tracto de salida del ventrículo izquierdo (TSVI) en ningún caso ${ }^{(13)}$.

Si bien los procedimientos VIR están en pleno desarrollo, los resultados hasta el momento no son tan favorables como los de VIVM, como surge del registro Reemplazo valvular mitral transcatéter (TMVR). Se trata de un registro internacional, observacional, sobre procedimiento VIV o VIR mitral. Un total de 248 pacientes fue tratado con reemplazo mitral transcatéter. El score STS medio fue de $8,9 \pm 6,8 \%$. El acceso transeptal fue utilizado en $33,1 \%$ de los casos y el transapical en el resto de la población. Se utilizaron válvulas expansibles por balón en 89,9\% de los casos. En 176 pacientes se realizó procedimiento VIVM y en 72 VIR mitral. El éxito se logró en 92,3\%, aunque fue mayor en el grupo VIV (96,0\%) en comparación con el grupo VIR $(83,3 \%)$, en el que se comprobó mayor necesidad de implante de una segunda prótesis y mayor necesidad de reintervención. Los gradientes transvalvulares posprocedimiento fueron similares en ambos grupos (6,4 $\pm 2,3$ mmHg vs $5,8 \pm 2,7 \mathrm{mmHg}, \mathrm{p}=0,17$ ) en tanto que la regurgitación mitral moderada o severa fue más frecuente en el grupo VIR; este grupo tuvo mayor frecuencia de sangrado mayor y de insuficiencia renal aguda $(11,1 \%$ vs $4,0 \%, p=0,03)$. La mortalidad global al año fue significativamente mayor en el grupo VIR comparado con el grupo VIV (28,7\% vs $12,6 \%, \log$ rank test $p=0,01)^{(14)}$. 
Características de las prótesis biológicas quirúrgicas disfuncionantes

Las prótesis valvulares biológicas quirúrgicas presentan algunas características favorables para el procedimiento VIV. El anillo y el stent de la bioprótesis constituyen estructuras rígidas o poco expansibles, dentro de las cuales la prótesis transcatéter puede fijarse adecuadamente. El anillo suele ser radioopaco, lo que facilita su visualización por radioscopía como referencia para la altura del implante y su forma es circular, lo que favorece la correcta aposición de la prótesis transcatéter y minimiza la posibilidad de insuficiencia residual periprotésica (interprotésica).

Un paso ineludible en el planeamiento de un procedimiento VIV es definir con precisión las características de la bioprótesis implantada quirúrgicamente, dado que cada modelo tiene particularidades estructurales y geométricas. Es mandatorio conocer marca, modelo y tamaño de la prótesis quirúrgica para poder elegir la prótesis transcatéter a implantar. En este sentido debe analizarse el protocolo operatorio siempre que sea posible. De todas maneras, las características radioscópicas de las bioprótesis permiten generalmente identificar el tipo de prótesis. Cada una de ellas tiene un diámetro interno, externo y algunas características que deben ser tenidas en cuenta antes y durante el procedimiento. La aplicación para teléfonos inteligentes VIV disponible, tanto para bioprótesis aórticas como mitrales, permite identificar el tipo de bioprótesis quirúrgica y de anillos de anuloplastia mitral en base a su aspecto radioscópico(15).

\section{Mecanismos de falla de las prótesis cardíacas biológicas}

La disfunción de las bioprótesis quirúrgicas puede ser bajo la forma de estenosis o de insuficiencia. Es importante conocer el mecanismo de la disfunción para realizar una adecuada opción terapéutica. La etiología de la disfunción de las prótesis biológicas puede ser consecuencia de mal funcionamiento de los velos protésicos o de las estructuras de soporte (anillo basal, stent protésico o ambos). En general, es secundaria al estrés mecánico ejercido sobre la prótesis, cuya repercusión es influenciada por características de diseño de la misma (por ejemplo, diseño estructural, tratamiento anticalcificación) y por características metabólicas del paciente (por ejemplo, insuficiencia renal)(16).

El deterioro estructural de las valvas es la causa más común de falla de las bioprótesis. Entre los mecanismos de disfunción protésica se encuentran el desgaste tisular, la calcificación valvar, la forma- ción de pannus, la trombosis y la endocarditis infecciosa. Esta última es una contraindicación para procedimientos VIV, al menos hasta que se asegure su cura infectológica.

La calcificación protésica tiende a ocurrir en los sitios de mayor flexión y estrés, que corresponden a la porción basal de los velos y a las comisuras protésicas, pero puede afectar a toda la extensión de una o más valvas.

El desarrollo de tejido inflamatorio, rico en fibroblastos en la interfase paciente-injerto, se conoce como pannus y está presente en mayor o menor grado en la mayoría de las prótesis. La formación marcada de pannus puede inducir disfunción protésica si se extiende a las cúspides protésicas y reduce su motilidad. La trombosis y la endocarditis infecciosa son causas menos comunes de disfunción protésica.

\section{Acceso para VIVM}

El acceso para procedimiento VIVM incluye el abordaje transapical, que es el más utilizado, el abordaje transeptal y el abordaje directo de la aurícula izquierda, usado infrecuentemente. El abordaje transeptal se realiza en forma completamente percutánea, en general a través de la vena femoral y aborda la aurícula izquierda a través de una punción del septum interauricular, que en general es guiada por ecocardiografía transesofágica. La utilización de este acceso es variable en diferentes series. En el registro TMVR el acceso transeptal se utilizó en el 33,1\% de los $\operatorname{casos}^{(14)}$, en tanto que en el Registro internacional de datos valve-in-valve (VIVID) se utilizó en el 16\%, aunque esta frecuencia ha aumentado desde $15 \%$ en el inicio del registro hasta $25,4 \%$ entre 2014$2015^{(17)}$.

El acceso transapical, aunque invasivo, ofrece características favorables para el abordaje mitral, tal como fue descrito y utilizado por Byk y colaboradores en el caso presentado en el número anterior de la RUC(10). El ápex cardíaco es anatómicamente cercano a la válvula mitral, lo que puede permitir un control más adecuado del dispositivo a implantar en comparación con el acceso transeptal que implica un recorrido transvenoso y un importante cambio de dirección intracardíaco para alcanzar el plano mitral. Esto hace que el implante sea algo más dificultoso por esta vía. Sin embargo, este acceso es menos invasivo, sin toracotomía ni lesión del ventrículo izquierdo. Se ha comprobado una mayor mejoría de la función ventricular izquierda en pacientes con disfunción sistólica previa tratados con VIVM por acceso transeptal en comparación con el 
acceso transapical ${ }^{(19)}$. Es previsible que en los próximos años aumente la utilización del acceso transeptal, tal como fue usado en el caso presentado por Merino y colaboradores ${ }^{(9)}$.

\section{Dispositivos utilizados para VIVM}

En general, las válvulas transcatéter utilizadas son expansibles por balón y desarrolladas para implante en válvulas aórticas nativas mediante procedimiento transcatéter o TAVI. La prótesis CoreValve (Medtronic) no es adecuada para esta topografía porque su alto perfil provocaría obstrucción al TSVI. Gran parte de la evidencia disponible se basa en el implante de prótesis Sapien (3 y XT, Edwards Lifesciences, Irvine, CA, USA), que en el registro VIVID representó el $90 \%$ de los dispositivos utiliza$\operatorname{dos}^{(18)}$.

Otras protésis expansibles por balón utilizadas incluyen la prótesis Melody (Medtronic, Minneapoliis, Mn, USA) y la Inovare (Braile Biomédica, São José do Rio Preto, Brazil).

Existe un rango reducido de tamaños de prótesis transcatéter para aplicación en VIVM, teniendo en cuenta el mayor diámetro del anillo mitral en comparación con el anillo aórtico. Esta consideración se basa en que las prótesis mitrales quirúrgicas miden 25 a $31 \mathrm{~mm}$ y que las más frecuentemente implantadas son las de 29 y $31 \mathrm{~mm}$. Por otro lado, la válvula Edwards Sapien de mayor diámetro disponible es de $29 \mathrm{~mm}$, lo que implica que podría ser inadecuada para un número significativo de casos.

\section{Elección del dispositivo a implantar}

La elección del dispositivo a implantar es de la mayor importancia para el éxito del procedimiento, tanto en lo que respecta al perfil o altura del dispositivo como a su diámetro. La altura del dispositivo se relaciona eventualmente con complicaciones como la obstrucción del TSVI en el implante mitral. El diámetro del dispositivo es fundamental para su correcta función y para evitar la migración de la prótesis. El implante de una prótesis transcatéter pequeña para la prótesis quirúrgica se acompañaría de riesgo de migración del dispositivo y de insuficiencia residual interprotésica, en tanto que una prótesis de mayor diámetro que el indicado determinaría una expansión inadecuada del dispositivo con disfunción de sus velos e insuficiencia protésica o estenosis, o ambas.

En general, la elección del dispositivo se basa en las especificaciones del fabricante de las características de la prótesis quirúrgica implantada. La aplicación valve-in-valve recomienda la prótesis trans- catéter adecuada para el procedimiento VIV según la bioprótesis disfuncionante, en base a una evaluación previa de la correspondencia entre cada tipo y tamaño de bioprótesis quirúrgica con las prótesis transcatéter disponibles ${ }^{(15)}$.

La imagenología cardiovascular juega un rol importante en la evaluación preprocedimiento. El estudio principal es la ecocardiografía transtorácica y transesofágica, que permite en primer lugar evaluar el tipo de la disfunción: estenosis, insuficiencia o ambas. En el caso de comprobarse insuficiencia debe definirse si esta es protésica o paraprotésica, dado que el procedimiento no está diseñado para tratar la insuficiencia paraprotésica, teniendo en cuenta que la nueva prótesis se implanta dentro de una bioprótesis rígida, lo cual no modifica el ambiente periprotésico. La ecocardiografía permite también definir el mecanismo de la disfunción protésica incluyendo pannus, calcificación, endocarditis (complicaciones periprotésicas, vegetaciones, etcétera) y trombosis.

La ecocardiografía tiene un rol muy importante para prevenir complicaciones del procedimiento VIV. Permite evaluar la magnitud y topografía de la calcificación que puede interferir con la expansión de la prótesis durante el implante y permite identificar características anatómicas asociadas con obstrucción del TSVI, como se señalará más adelante.

La tomografía multicorte permite evaluar las características anatómicas reales de la bioprótesis quirúrgica, incluyendo el tamaño interno del anillo y grado y distribución de la calcificación. Es posible realizar una simulación del resultado del implante para elegir el mejor dispositivo, previendo complicaciones. Una descripción detallada del rol de la tomografía en VIVM se encuentra en la revisión de Guerrero y colaboradores ${ }^{(19)}$.

\section{Complicaciones del procedimiento VIVM: cómo prevenirlas}

En general, el mejor tratamiento de las complicaciones es su prevención en base a un planeamiento exhaustivo y detallado del procedimiento. Las complicaciones más relevantes y específicas incluyen:

\section{a) Malposición de la prótesis}

La posición inadecuada de la válvula durante VIVM se ha documentado en el 7\% de los casos aproximadamente en el registro VIVID ${ }^{(18)}$. Esto se ha asociado con el implante dentro de bioprótesis quirúrgicas con estructura poco radioopaca, que dificulta ubicar referencias adecuadas para el implante. La monitorización con ecocardiografía transesofágica intraprocedimiento es fundamental en estos casos. Por otro lado, se ha documentado la malposición tardía 
de la prótesis, que implica la migración hacia la aurícula izquierda típicamente algunas semanas después del implante. Esta complicación se asocia a un grado insuficiente de sobredimensionamiento de la prótesis transcatéter con respecto a la bioprótesis quirúrgica (oversizing). Algunos autores preconizan que el oversizing debe ser mayor en VIVIM que en TAVI, pero no existe acuerdo sobre la correcta magnitud del mismo ${ }^{(15)}$.

\section{b) Obstrucción del tracto de salida del ventrículo izquierdo}

La nueva prótesis implantada dentro de la preexistente podría protruir en el TSVI, generando obstrucción del mismo. Variables asociadas con la aparición de esta complicación incluyen la relación entre el plano mitral y el aórtico (cuanto más alineados, más riesgo de obstrucción), el perfil de la prótesis (cuanto mayor, más riesgo de obstrucción), la profundidad del implante (cuanto más hacia el lado ventricular se haga el implante, mayor el riesgo de obstrucción), espesor del septum interventricular (cuanto más protruyente sea el septum, mayor riesgo de obstrucción en el TSVI). En todos los casos debe evitarse esta complicación en base a estudios de imágenes como ecocardiograma y/o tomografía multicorte cardíaca; esta última permite realizar una simulación adecuada del efecto del implante sobre el TSVI ${ }^{(20)}$.

\section{c) Gradiente transmitral elevado posprocedimiento}

Se ha recomendado considerar gradientes trasmitrales posprocedimiento mayores de $5 \mathrm{mmHg}$ como fracaso del procedimiento ${ }^{(21)}$. Otros autores han planteado definiciones menos exigentes teniendo en cuenta que se han comprobado gradientes mayores de $10 \mathrm{mmHg}$ en el $27 \%$ de los casos del registro VIVID $^{(17)}$.

De todas formas, la incidencia de gradientes elevados pos VIVM es menor que la comprobada en VIV aórtico, en especial cuando en esta topografía la bioprótesis quirúrgica es menor de 23 $\mathrm{mm}^{(22)}$

\section{d) Insuficiencia mitral residual posprocedimiento}

La insuficiencia mitral pos VIVM es infrecuente y se ha reportado en el rango de $2 \%$ a $3 \%$ en el registro VIVID. En general, la aposición de la nueva prótesis es correcta y no se comprueba insuficienca interprótesica si se eligió el tamaño adecuado de prótesis. Cuando se comprueba insuficiencia mitral significativa debe pesquizarse una fuga paraprotésica de la prótesis quirúrgica no advertida previamente.

\section{Conclusiones}

La disfunción de las bioprótesis quirúrgicas constituye un problema clínico de frecuencia creciente. Los procedimientos VIV representan una alternativa menos invasiva y de menor riesgo que la reintervención quirúrgica en pacientes de alto riesgo operatorio. Un adecuado conocimiento de la bioprótesis, de su mecanismo de falla, de los dispositivos y técnicas adecuadas para el implante, son prioritarios para el éxito del procedimiento. Los procedimientos VIV y VIR representan un nuevo campo de desarrollo que extiende las fronteras de la cardiología intervencionista estructural y que debemos incorporar en nuestra práctica cardiológica.

\section{Bibliografía}

1. Hammermeister K, Sethi GK, Henderson WG, Grover FL, Oprian C, Rahimtoola SH. Outcomes 15 years after valve replacement with a mechanical versus a bioprosthetic valve: final report of the Veterans Affairs randomized trial. J Am Coll Cardiol 2000;36 (4):1152-8.

2. Piazza N, Bleiziffer S, Brockmann G, Hendrick R, Deutsch MA, Opitz A, et al. Transcatheter aortic valve implantation for failing surgical aortic bioprosthetic valve: from concept to clinical application and evaluation (part 1). JACC Cardiovasc Interv 2011;4(7):721-32. doi: 10.1016/j.jcin.2011.03.016.

3. Baumgartner H, Falk V, Bax JJ, De Bonis M, Hamm C, Holm PJ, et al. 2017 ESC/EACTS Guidelines for the management of valvular heart disease. Eur Heart J. 2017;38(36):2739-91.

4. Gammie JS, Sheng S, Griffith BP, Peterson ED, Rankin JS, O'Brien SM, et al. Trends in mitral valve surgery in the United States: results from the Society of Thoracic Surgeons Adult Cardiac Surgery Database. Ann Thorac Surg. 2009;87(5):1431-7; discussion 1437-9.

doi: 10.1016/j.athoracsur.2009.01.064.

5. Cohn LH, Aranki SF, Rizzo RJ, Adams DH, Cogswell KA, Kinchla NM, et al. Decrease in operative risk of reoperative valve surgery. Ann Thorac Surg.1993;56(1):15-20; discussion 20-1.

6. Vogt PR, Brunner-La Rocea $\mathbf{H}$, Sidler P, Zünd G, Truniger K, Lachat M, et al. Reoperative surgery for degenerated aortic bioprostheses: predictors for emergency surgery and reoperative mortality. Eur J Cardiothorac Surg.2000;17(2):134-9.

7. Akins CW, Buckley MJ, Daggett WM, Hilgenberg AD, Vlahakes GJ, Torchiana DF, et al. Risk of reoperative valve replacement for failed mitral and aortic bioprostheses. Ann Thorac Surg 1998;65(6):1545-51; discussion 1551-2. 
8. Maganti M, Rao V, Armstrong S, Feindel CM, Scully HE, David TE, et al. Redo valvular surgery in elderly patients. Ann Thorac Surg 2009;87(2): 521-5. doi: 10.1016/j.athoracsur.2008.09.030

9. Merino Rajme JA, Escutia Cuevas HH, Alcántara Meléndez MA, Fernández Ceseña E, García García JF, Morales Portano JD, et al. Implantación percutánea transeptal de válvula protésica mitral (valve-in-valve). Reporte de caso. Rev Urug Cardiol 2017;32(2):191-6.

10. Byk B, Cedrés G, Soca G, Marichal A, Paganini JJ, Trujillo P, et al. Implante de prótesis en posición mitral mediante procedimiento valve-in-valve por vía transapical. Reporte de caso. Rev Urug Cardiol 2017;32(2):196-7.

11. Cheung A, Webb JG, Wong DR, Ye J, Masson JB, Carere RG, et al. Transapical transcatheter mitral valve-in-valve implantation in a human. Ann Thorac Surg 2009;87(3):e18-20.

doi: 10.1016/j.athoracsur.2008.10.016.

12. Cheung A, Webb JG, Barbanti M, Freeman M, Binder RK, Thompson C, et al. 5-year experience with transcatheter transapical mitral valve-in-valve implantation for bioprosthetic valve dysfunction. J Am Coll Cardiol 2013;61 (17): 1759-66. doi: 10.1016/j.jacc.2013.01.058.

13. Gaia DF, Braz AM, Simonato M, Dvir D, Breda JR, Ribeiro GC, et al. Mitral implant of the Inovare transcatheter heart valve in failed surgical bioprostheses: a novel alternative for valve-in-valve procedures. Interact Cardiovasc Thorac Surg 2017;24(4): 514-520. doi: 10.1093/icvts/ivw368

14. Yoon SH, Whisenant BK, Bleiziffer S, Delgado V, Schofer N, Eschenbach L, et al. Transcatheter mitral valve replacement for degenerated bioprosthetic valves and failed annuloplasty rings. J Am Coll Cardiol. 2017;70(9):1121-31.

doi: 10.1016/j.jacc.2017.07.714

15. Bapat V. Valve-in-valve apps: why and how they were developed and how to use them. Euro Interven- tion 2014;10 (Suppl. U):U44-51.

doi: 10.4244/EIJV10SUA7

16. Roselli EE, Smedira NG, Blackstone EH. Failure modes of the Carpentier-Edwards pericardial bioprosthesis in the aortic position. J Heart Valve Dis 2006;15(3):421-7 ; discussion 427-8

17. Dvir D, Webb J. Mitral valve-in-valve and valve-in-ring: technical aspects and procedural outcomes. Euro Intervention 2016;12(Suppl Y):Y93-Y96. doi: 10.4244/EIJV12SYA25

18. Dvir D. Transseptal instead of transapical valve implantation: making mitral great again? JACC Cardiovasc Interv 2016;9(11):1175-7.

doi: $10.1016 /$ j.jcin.2016.04.006.

19. Guerrero M, Salinger M, Pursnani A, Pearson P, Lampert M, Levisay J, et al. Transseptal transcatheter mitral valve-in-valve: A step by step guide from preprocedural planning to postprocedural care. Catheter Cardiovasc Interv 2017. doi: 10.1002/ccd.27128. [Epub ahead of print]

20. Blanke P, Naoum C, Dvir D, Bapat V, Ong K, Muller D, et al. Predicting LVOT Obstruction in Transcatheter Mitral Valve Implantation: Concept of the Neo-LVOT. JACC Cardiovasc Imaging 2017;10(4):482-485. doi: 10.1016/j.jcmg.2016.01.005

21. Stone GW, Adams DH, Abraham WT, Kappetein AP, Généreux P, Vranckx P, et.al. Clinical trial design principles and endpoint definitions for transcatheter mitral valve repair and replacement: Part 2: Endpoint definitions: a consensus document from the Mitral Valve Academic Research Consortium. J Am Coll Cardiol 2015;66(3):308-21. doi: 10.1016/j.jacc.2015.05.049

22. Ye J, Cheung A, Yamashita M, Wood D, Peng D, Gao M, et al. TranscatheteaAortic and mitral valve-in-valve implantation for failed surgical bioprosthetic valves: An 8-year Single-Center Experience. JACC Cardiovasc Interv. 2015;8(13):1735-44. doi: 10.1016/j.jcin.2015.08.012. 